\title{
VALOR DE TRANSACCIÓN DEL SUELO AGRÍCOLA EN EL SUR DE CHILE: PERÍODO 1991 A 2000¹
}

\author{
AGRICULTURAL SOIL TRANSACTION VALUE IN SOUTHERN CHILE: \\ FROM 1991 TO 2000
}

Víctor H. Moreira López ${ }^{2}$; Daniela L. Ruiz Carreño ${ }^{3}$

\begin{abstract}
RESUMEN
Con el objetivo de realizar un estudio sobre la evolución que ha tenido el precio del suelo agrícola de la Décima y Decimocuarta Regiones en el período 1991-2000, se recopiló y analizó la información perteneciente a los Conservadores de Bienes Raíces de las provincias de Valdivia, Lago Ranco, Osorno, Llanquihue y Chiloé. Se observó que los suelos agrícolas han experimentado un incremento real de un 38\% en el precio informado entre 1991 y 2000. En las provincias de Valdivia y Lago Ranco la comuna de Lago Ranco posee los mayores precios. En la Provincia de Osorno la comuna de San Juan de la Costa presenta los menores precios de suelos agrícolas, y la comuna de Osorno se encuentra en el otro extremo con los mayores valores. En la Provincia de Llanquihue las comunas analizadas no presentan diferencias significativas. Por último, en la Provincia de Chiloé se presentan los mayores precios en la comuna de Castro. El resto de las comunas no presenta diferencias significativas.
\end{abstract}

Palabras clave: Valor de transacción, plusvalía, suelos agrícolas, sur de Chile.

\section{ABSTRACT}

In order to study the evolution that the agricultural soil price of the tenth and fourteenth regions has had between 1991-2000, it was collected and analyzed the information that belong to the Property Sold Records (Conservadores de Bienes Raices) from the following Provinces: Valdivia, Lago Ranco, Osorno, Llanquihue and Chiloé. It was observed that the agricultural soil price has an increase of 38\% between 1991 and 2000. In the Provinces of Valdivia and Lago Ranco, the Lago Ranco Community shows the highest soil price. In the Osorno Province, the San Juan de la Costa Community has the lowest agricultural soil price. In the Llanquihue Province, all the Communities have no significant differences in the soil price. Finally, in the Chiloe Province, the Castro Community evidences the highest soil price, and the prices for the other Communities do not have significant differences.

Key words: transaction value, capital gain, agrarian soil, Southern Chile.

\section{INTRODUCCIÓN}

El suelo es considerado un recurso no depreciable, es decir, a medida que transcurre el tiempo éste no pierde su valor por concepto de uso u obsolescencia, sino que por el contrario sufre un aumento real, denominado plusvalía. La variación del precio del suelo se puede deber a variados factores, entre los cuales se encuentran: la rentabilidad de la explotación agrícola (Troncoso y Calderón, 2000; Guadalajara, 1996), la ubicación del predio (Peralta, 1994; Morandé y Soto, 1992; Samaniego, 2001), vías de acceso (Guadalajara, 1996), el tipo de suelo (Guadalajara, 1996), el tamaño (Morales et al., 1999; Samaniego, 2001; Schonhaut, 2000), existencia o no de riego (Meneses, 1999; Morales et al., 1999; Schonhaut, 2000), entre otros, los cuales pueden tener mayor o menor relevancia en el precio final del suelo.

1 La recolección de información fue parcialmente financiada por Proyecto S-200070, Dirección de Investigación y Desarrollo (DID) de la Universidad Austral de Chile (UACh). Este trabajo está basado en parte en los resultados generados en una tesis de grado de la Facultad de Ciencias Agrarias de la UACh.

2 Universidad Austral de Chile, Facultad de Ciencias Agrarias. Independencia 641, casilla 567, Valdivia, Chile. Email: vmoreira@uach.cl

3 Colon 810, Los Ángeles, Chile. Email: danielaruizcarreno@gmail.com

Fecha de Recepción: 18 Abril 2007

Fecha de Aceptación: 06 Agosto 2007 
El proceso de valuación consiste en el análisis sistemático de los factores que influyen en el valor de una propiedad (Dobner, 1989). El precio que se da a la tierra en una transacción no corresponde precisamente a su valor comercial, sino a una combinación del precio de distintos tipos de bienes (Morandé y Soto, 1992). Según Peralta (1994), el valor de las propiedades agrícolas se encuentra relacionado con la capacidad de producción de sus tierras, su localización respecto al mercado consumidor y de abastecimiento.

El precio del suelo agrícola se encuentra influenciado por factores de calidad intrínseca, como es la calidad del suelo y factores específicos que también pueden afectar el valor comercial del suelo, como son: tipo de riego, tipo de clima y distancia a caminos principales (Morandé y Soto, 1992).

En el país se han realizado variados estudios para determinar el comportamiento del precio del suelo a través de los años, pero este es el primer análisis del comportamiento de los precios de los suelos transados en las provincias de Valdivia, Lago Ranco, Osorno, Llanquihue y Chiloé, basándose en información de los Conservadores de Bienes Raíces (CBR). El objetivo general de este trabajo es analizar la evolución que ha tenido el valor del suelo agrícola en el período 1991-2000, en cinco provincias de la Décima y Decimocuarta Regiones.

\section{MATERIALES Y MÉTODOS}

El universo de información son los registros que los CBR de la Décima y Decimocuarta Regiones poseen de las transacciones de suelo agrícola en el período 1991-2000. Los antecedentes utilizados corresponden a registros de transacciones de predios con una superficie mayor a 0,5 ha, y que fuesen utilizados en actividades silvoagropecuarias. Es decir, se excluyeron las parcelas de agrado, los predios de uso exclusivamente industrial, los predios en zonas urbanas y los predios con uso forestal.

El universo de estudio incluye $17 \mathrm{CBR}$ (Cuadro 1). Para la selección de los CBR en cada una de las provincias se consideró la superficie agrícola que éstos abarcan. El criterio de selección se basó en usar la información de aquellos CBR que abarcan al menos el $40 \%$ de la superficie agrícola total de cada provincia. Adicionalmente, es importante mencionar que no existe una estandarización en la forma y tipo de antecedentes que se registran en los CBR de las regiones en estudio, lo que implica que al no estar disponible la información requerida para el presente estudio se procedió a seleccionar el siguiente CBR con mayor superficie agrícola.

Para saber la superficie que abarca cada CBR por comuna se recurrió a los registros del SII, de donde se obtuvo la superficie agrícola de cada una de las comunas. En el Cuadro 1 se destacan los CBR finalmente incluidos en el estudio.

La información se obtuvo a través de una encuesta diseñada con el objetivo de recolectar la información primaria requerida, recopilando las transacciones de predios agrícolas realizadas en las cinco provincias de la Décima y Decimocuarta Regiones. La información recopilada fue: identificación del predio: propietario, rol de avalúo fiscal e inscripción de dominio; ubicación: provincia y comuna y superficie total.

Adicionalmente se realizó una encuesta secundaria. Este instrumento se empleó en diferentes etapas de la investigación. Inicialmente se consultó la opinión de expertos (corredores de propiedades y tasadores calificados), quienes ayudaron a validar la pertinencia de realizar un estudio basado en la información de los CBR o de los registros de las transacciones realizadas por corredores de propiedades. Las respuestas fueron categóricas y respaldaron la idea de obtener información de los CBR. Lo anterior se justifica en diferentes aspectos. Primero, en la zona Sur de Chile no existe una organización de corredores de propiedades que acumule la información de las transacciones, tal como ocurre en la zona Central del país. Al no existir un ente que recopile la información de los corredores de propiedades no hay una estandarización de los registros, es decir, cada corredor de la zona Sur posee un formato diferente, así como un sistema de registro distinto, lo que hace muy compleja la alternativa de recopilar la información en forma directa desde sus registros.

Por otra parte, la encuesta secundaria se aplicó a profesionales expertos en el tema (tasadores y asesores agrícolas) de la Décima y Decimocuarta Regiones, teniendo como objetivo el determinar rangos entre los cuales se encontraban los precios de suelos agrícolas de las provincias analizadas, de manera de disponer de mayores antecedentes para una apropiada selección de las transacciones a ser analizadas.

Para recopilar la información requerida se procedió a viajar a las "ciudades asiento" de cada uno 
Cuadro 1

Conservador de Bienes Raíces (CBR) correspondientes a las provincias de Valdivia, Osorno, Llanquihue y Chiloé

\begin{tabular}{|c|c|c|}
\hline Provincia & Ubicación del CBR & Comunas que abarca \\
\hline \multirow{7}{*}{ Valdivia } & Valdivia $^{1}$ & Valdivia y Corral. \\
\hline & San José de la Mariquina & San José de la Mariquina, Lanco y Mafil. \\
\hline & Los Lagos & Los Lagos y Futrono. \\
\hline & Panguipulli $^{1}$ & Panguipulli. \\
\hline & La Unión & La Unión. \\
\hline & Paillaco & Paillaco. \\
\hline & Río Bueno ${ }^{1,2}$ & Río Bueno y Lago Ranco. \\
\hline \multirow{2}{*}{ Osorno } & Osorno $^{1}$ & Osorno, San Juan de la Costa, Puyehue, Puerto Octay y San Pablo. \\
\hline & Río Negro & Río Negro y Purranque. \\
\hline \multirow{4}{*}{ Llanquihue } & Puerto Varas & Puerto Varas, Llanquihue y Fresia. \\
\hline & Los Muermos & Los Muermos. \\
\hline & Puerto Montt ${ }^{1}$ & Puerto Montt, Cochamó y Hualaihue. \\
\hline & Calbuco & Calbuco. \\
\hline \multirow{4}{*}{ Chiloé } & Achao & Achao, Curaco de Vélez, Quinchao. \\
\hline & Castro $^{1}$ & Castro, Chonchi, Dalcahue, Puqueldón y Queilén. \\
\hline & Ancud & Ancud y Quemchi. \\
\hline & Quellón ${ }^{1}$ & Quellón. \\
\hline
\end{tabular}

1 CBR incluidos en el análisis.

2 Según la actual distribución geopolítica de Chile, la comuna de Lago Ranco pertenece a la provincia del mismo nombre. Sin embargo, al momento de realizar la recopilación de datos, la comuna de Lago Ranco pertenecía a la Provincia de Valdivia. De esta manera, para el presente trabajo los datos recopilados de la Comuna de Lago Ranco están incluidos como parte de la Provincia de Valdivia.

Fuente: Información recopilada por los autores en visitas realizadas a los CBR en estudio.

de los CBR seleccionados, revisando los Libros de Dominio o de Propiedad correspondientes al período que abarca desde 1991 al 2000. Dentro de éstos se revisaron todas las transacciones rotuladas como compraventa, seleccionando aquellas que cumplieran con los requisitos antes mencionados.

Todas las cifras monetarias se actualizaron (Valor Actual) a diciembre de 2006, para lo cual se utilizó la variación promedio del IPC para el año de transacción ( $\mathrm{IPC}_{\mathrm{t}}$ ), la variación del IPC a diciembre de $2006\left(\right.$ IPC $\left._{\text {dic2006 }}\right)$ y el Valor de transacción, a través del siguiente cálculo:

$$
\text { Valor Actual }=\frac{\mathrm{IPC}_{\mathrm{t}} * \text { Valor de transacción }}{\mathrm{IPC}_{\mathrm{dic} 2006}}
$$

Después de determinar el valor unitario (por hectárea) de cada uno de los predios registrados se procedió a realizar una depuración de la información, considerando la información obtenida con la encuesta secundaria. Los profesionales expertos informaron que los rangos de precios de suelos agrícolas transados en la Décima y Decimocuarta Regiones fluctúan entre \$100.000/ha y \$3.500.000/ha (moneda a septiembre de 2001), por lo que estos precios fueron considerados como la base y el techo para la depuración de datos.

\section{RESULTADOS Y DISCUSIÓN}

La selección de los CBR utilizados para este estudio no estuvo exenta de dificultades. El principal problema estuvo en el hecho de que no todos registran la información requerida en forma completa y de fácil acceso. Ante la ausencia de información básica como, por ejemplo, la superficie transada o el valor de transacción, se procedió a descartar dicho CBR y utilizar datos de otro Conservador, elegido según el criterio antes detallado. Esta circunstancia particular se presentó en la Provincia de Llanquihue, 
en la cual sólo se pudo utilizar información del CBR de Puerto Montt, donde se registran los datos esenciales para el presente estudio.

Del total de CBR que existen en la Décima y Decimocuarta Regiones se recopiló la información en siete. De ellos se logró obtener: la fecha de inscripción de la propiedad, el tamaño del predio transado, la comuna a la cual pertenecía, el valor de transacción y el rol de avalúo fiscal.

Entre los principales resultados del presente estudio, la Figura 1 refleja el valor de transacción del suelo agrícola para el período 1991-2000 en cinco provincias de la Décima y Decimocuarta Regiones. La Figura 1 contiene en forma clara los precios promedios de los suelos agrícolas para las cinco provincias en análisis.

El valor de las transacciones de suelos agrícolas entre 1991-2000 en las cinco provincias de la Décima y Decimocuarta Regiones muestra un aumento real en el precio unitario de un $38 \%$ (en 10 años).

En el año 1992 el precio del suelo sufrió un pequeño descenso, recuperándose a partir de 1993 hasta 1995. Desde 1996 hasta 1998 el precio del suelo continúa aumentando, pero a una menor tasa. En el año 1999 se produce una segunda disminución en el precio del suelo agrícola, después de lo cual éste nuevamente comienza a aumentar.

Las variaciones que sufren los precios de los terrenos agrícolas se deben a una variada gama de factores. En el estudio de la Pontificia Universidad Católica de Chile, Departamento de Economía Agraria (1979), se señala que la rentabilidad del sector agrícola puede afectar el valor de la tierra, por lo que éste sería un elemento importante a considerar. Tal como fue descrito en la metodología, la rentabilidad del sector agrícola no está entre las variables recopiladas. Sin embargo, el Producto Interno Bruto (PIB) es una medida indirecta de la intensidad económica del sector agrícola, lo que podría ser usado como una aproximación de la rentabilidad agrícola. El PIB mide el flujo total de bienes y servicios que produce la economía durante un determinado período (Bannock, 1990), y en el caso del sector silvoagropecuario a nivel nacional, éste ha aumentado en un 38,8\% desde el año 1990

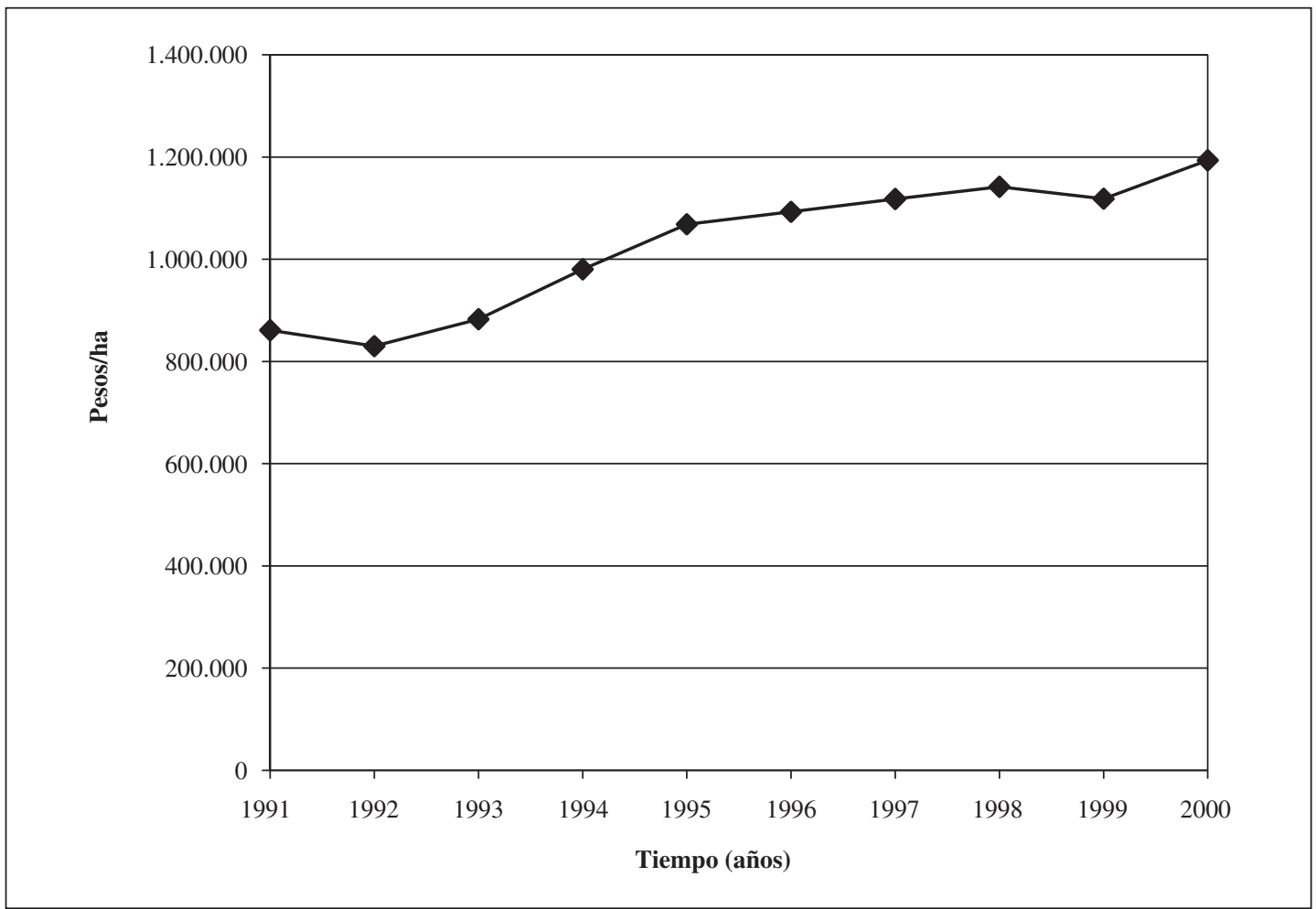

Figura 1. Evolución de los precios de los suelos agrícolas correspondientes a cuatro provincias de la Décima y Decimocuarta Regiones para el período 1991-2000 (cifras en moneda de diciembre de 2006).

Fuente: Resultados recopilados y procesados por los autores en visitas realizadas a los CBR en estudio. 
al 2001 (Oficina de Estudio y Políticas Agrarias, ODEPA, 2001).

En el ámbito nacional las exportaciones en general han aumentado casi al doble desde 1990 hasta 1999. En el caso de los productos silvoagropecuarios y pesca, las exportaciones han aumentado alrededor de un 50\%, lo que también conlleva un mayor interés por la actividad agrícola, e indirectamente puede afectar el valor del suelo. En la Décima y Decimocuarta Regiones las exportaciones crecieron en un porcentaje similar al expuesto anteriormente, lo que podría tener algún efecto en el precio de los suelos de las Regiones en estudio, ya que es esperable que los productos silvoagropecuarios estén considerados como una alternativa rentable para los productores de la zona (Ministerio de Planificación y Cooperación, MIDEPLAN, 2000). Esto se ve corroborado por la balanza comercial para la Décima y Decimocuarta Regiones, la que experimentó un crecimiento en los productos silvoagropecuarios, con un claro aumento desde el año 1990 al 2000, con valores de 2.029 millones de dólares a 4.986 millones de dólares, respectivamente (ODEPA, 2001).

Estadísticas del catastro frutícola de CIRENCORFO de la temporada 1990-1991 (CIREN-CORFO, 1991) señalan que en las Regiones Décima y Decimocuarta existían alrededor de 116 hectáreas de manzanos rojos y 48 hectáreas de manzanos verdes como huertos industriales; el Censo 19961997 registró 416 hectáreas de manzanos rojos y 255 hectáreas de manzanos verdes, además de 11.466 hectáreas de huertos caseros (principalmente manzanos), por lo que se podría considerar este rubro como un negocio atractivo para la zona. Además, es importante señalar que se ha producido un interesante impulso en las plantaciones de esta especie en la Décima y Decimocuarta Regiones, lo que podría generar un aumento en los precios de los suelos agrícolas de las cinco provincias en estudio (Corporación de Fomento de la Producción, CORFO, 1998).

Tal como fuese mencionado, los factores que determinan el valor del suelo son variados y pueden afectarlo con mayor o menor intensidad, dependiendo de cuál se trate. Entre los factores analizados en esta investigación se encuentran: la ubicación del predio (provincia, comuna). Peralta (1994) señala que la ubicación es un elemento importante a considerar a la hora de comprar un predio. Éste involucra una serie de factores, entre los cuales se encuentran la ubicación geográfica, la cercanía a mercados de consumo de productos agrícolas, las vías de acceso tanto al predio como a caminos principales. En el presente estudio sólo se analiza la localización a nivel provincial y comunal, ya que fue la única información que se pudo recopilar de los registros de los CBR.

El valor promedio por hectárea obtenido para cada una de las cinco provincias refleja que la Provincia de Chiloé posee los menores valores unitarios, alcanzando un valor promedio de $\$ 791.337 / \mathrm{ha}$, seguida por las provincias de Osorno (promedio de \$1.056.862/ha), luego Valdivia (\$.067.359/ha), y, por último, Llanquihue (\$1.157.239/ha). Sin embargo, Chiloé es la única provincia que presenta diferencias significativas en relación al resto de las provincias.

Como información complementaria se puede señalar que de las cinco provincias analizadas Chiloé es aquella que se encuentra más alejada de los centros de mercado de productos agrícolas, ya que geográficamente se localiza en la Isla Grande de Chiloé, ubicada al Sur de la Décima Región. De esta forma se podría suponer que las vías de acceso a la Isla constituyen una limitante y que podría influenciar un mayor costo de transporte de los productos agrícolas hacia el resto del país. En cambio, las provincias de Valdivia, Osorno y Llanquihue se encuentran ubicadas en la parte continental de Chile y están conectadas a través de la Ruta 5 Sur, lo que facilitaría el acceso a cualquier ciudad del país.

En la Figura 2 se presentan los valores unitarios promedios por comuna. Se incluyen las comunas de Panguipulli, Valdivia, Corral, Lago Ranco, Río Bueno, Osorno, San Pablo, San Juan de la Costa, Puerto Octay, Puyehue, Puerto Montt, Cochamó, Dalcahue, Castro, Chonchi, Queilén, Puqueldón y Quellón, para el período 1991-2000.

De las 18 comunas analizadas se observa que San Juan de la Costa (Provincia de Osorno) y Queilén (Provincia de Chiloé) presentan los menores valores unitarios promedio para el período 1991-2000. En el otro extremo se encuentran las comunas de Osorno (Provincia de Osorno) y Lago Ranco (Provincia de Lago Ranco), las que presentaron los mayores valores unitarios transados para el período en estudio.

Al realizar un análisis más detallado por comunas en cada provincia se puede indicar que en el caso 


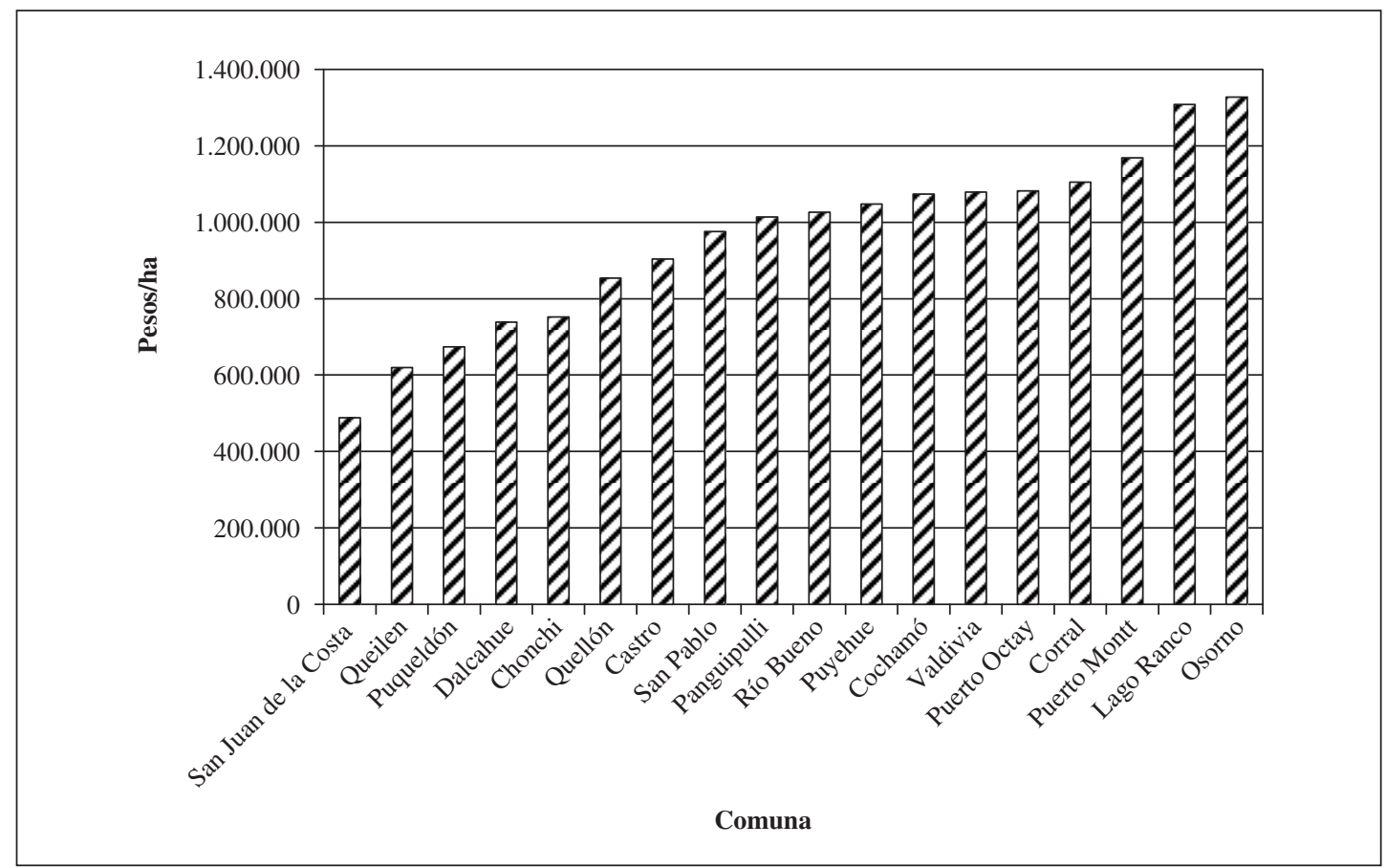

Figura 2. Precios unitarios promedio por Comuna, para el período 1991-2000 (cifras en moneda de diciembre de 2006). Fuente: Resultados recopilados y procesados por los autores en visitas realizadas a los CBR en estudio.

de las comunas de Panguipulli, Valdivia, Corral, Lago Ranco y Río Bueno, correspondientes a la Provincia de Valdivia, sólo Lago Ranco presenta valores estadísticamente superiores a las otras comunas, con un precio promedio de \$ 1.307.940/ha. El resultado anterior no presenta una justificación simple, lo que obliga a realizar futuros esfuerzos encaminados para este propósito.

En la Provincia de Osorno se analizan las comunas de San Pablo, San Juan de la Costa, Puyehue, Puerto Octay y Osorno. En esta provincia se detectó una marcada diferencia entre las comunas. Es así como San Juan de la Costa corresponde a la comuna que presenta los precios más bajos, alcanzando un valor promedio de $\$ 488.260 /$ ha, lo cual se complementa con el hecho de que San Juan de la Costa es considerada como una de las comunas más pobres de la Provincia de Osorno, lo que afectaría directamente el precio de los suelos agrícolas. En el otro extremo se encuentra la Comuna de Osorno, que alcanza valores promedio de $\$ 1.327 .806 /$ ha. En esta comuna se ubica la capital provincial, lo que podría justificar el hecho de que presente los mayores valores unitarios de transacción de suelos agrícolas. Además, se debe considerar que en ella se encuentran los principales mercados para los productos agrícolas. El resto de las comunas analizadas (San Pablo, Puyehue y Puerto Octay) no presentaron diferencias significativas.

En la Provincia de Llanquihue se analizaron dos comunas: Puerto Montt y Cochamó. Estas comunas no presentan diferencias significativas en los valores de los predios transados.

En relación a los valores unitarios promedio para las comunas de Queilén, Puqueldón, Dalcahue, Chonchi, Quellón y Castro, correspondientes a la Provincia de Chiloé, Castro es la comuna que presenta los mayores valores unitarios de suelos transados, lo que era esperable, ya que en Castro se encuentra la capital provincial y la ciudad con mayor importancia económica dentro de la Isla Grande de Chiloé. Además, Castro es considerado como el principal centro turístico de la Isla, circunstancia que podría influir positivamente en el precio de los suelos. Las comunas de Queilén, Puqueldón, Dalcahue, Chonchi y Quellón no presentan diferencias significativas en los valores unitarios de los suelos agrícolas transados. 


\section{LITERATURA CITADA}

BANNOCK, G. 1990. Diccionario de economía. México. Trillas. 391 p.

CENTRODE INFORMACIÓNDE RECURSOS NATURALES (CIREN) Y CORPORACIÓN DE FOMENTO DE LA PRODUCCIÓN. 1991. Catastro Frutícola Nacional IX y X Regiones. 129 p.

CORPORACIÓN DE FOMENTO DE LA PRODUCCIÓN (CORFO). 1998. Sector agropecuario nacional. Resultados de los talleres de planificación estratégica regional. Chile. $386 \mathrm{p}$.

DOBNER, H. 1989. La valuación de predios rurales. Concepto S.A. México. 315 p.

GUADALAJARA, N. 1996. Valoración agraria. Casos prácticos Mundi-Prensa. España. 277 p.

MENESES, E. 1999. Evolución del precio de los suelos agrícolas en las Regiones VII, VIII, IX en el período 1988-1997. Tesis Lic. Agr. Valparaíso. Universidad Católica, Facultad de Agronomía. 61 p.

MINISTERIO DE PLANIFICACIÓN Y COOPERACIÓN, DIRECCIÓN NACIONAL DE ADUANAS, DIVISIÓN DE PLANIFICACIÓN REGIONAL, MIDEPLAN, 2000. Exportaciones regionales del año 1998. <http://www. mideplan.cl/sitio/Sitio/publicaciones/htm/publicaciones. htm>. (09 abr. 2002).

MORALES, H., DÍAZ, J. Y BRAVO-URETA, B. 1999. Estudio del valor del suelo en las zonas Centro y Centro
Sur de Chile. Trabajo presentado en el IV Encuentro de Economistas Agrarios. 15 p.

MORANDÉ, F. Y SOTO, R. 1992. Una nota sobre la construcción de series de precios de activos reales: tierra y casas en Chile (1976-1989). Revista de Análisis Económico 7 (2): 169-177.

OFICINA DE ESTUDIOS Y POLÍTICAS AGRARIAS, ODEPA, 2001. Estadísticas agropecuarias 1990-2000. < http:///www.odepa.cl >. (28 mar. 2002).

PERALTA, J. 1994. Tasaciones: Contabilidad y tributación agrícola. JP. Chile. 192 p.

PONTIFICIA UNIVERSIDAD CATÓLICA DE CHILE, DEPARTAMENTO DE ECONOMÍA AGRARIA DE LA UNIVERSIDAD CATÓLICA. 1979. El precio de la tierra en Chile. Panorama Económico de la Agricultura 6: 3-7.

SAMANIEGO, S. 2001. Avalúos para la administración pública, avalúo de terrenos agropecuarios. <http://www.cabin.gob. $\mathrm{mx} /$ aniversario/memoria3.htm// >. (09 dic. 2001).

SCHONHAUT, D. 2000. Análisis de los precios de la tierra agrícola en Chile entre los años 1978-1998. Tesis Lic. Agr. Santiago. Pontificia Universidad Católica de Chile, Facultad de Agronomía e Ingeniería Forestal. 79 p.

TRONCOSO, J. Y CALDERÓN, J. 2000. Evolución del precio de la tierra y de la rentabilidad de la agricultura en el período 1983-1996. Panorama Socioeconómico 18 (21): 27-45. 
